Ann. Zootech., I972, 21 (I), 5-27.

\title{
EMPLOI DU TEMPS DES TROUPEAUX DE VACHES-MÈRES ET DE LEURS VEAUX SUR LES PÂTURAGES D'ALTITUDE DE L'AUBRAC
}

\author{
M. PETIT \\ Station de Recherches sur l'Élevage des Ruminants, \\ Centre de Recherches de Clermont-Ferrand, I. N. R. A., \\ 63 - Saint-Genès-Champanelle
}

\section{RÉSUMÉ}

On a enregistré l'emploi du temps (pâturage, tétée, station couchée, déplacements) de 6 troupeaux de 29 à 82 vaches et de leurs veaux sur des pâturages d'altitude de l'Aubrac (tabl. I) : 4 troupeaux de vaches allaitant leurs veaux en liberté ; un troupeau de vaches traites à la main 2 fois par jour; un troupeau de vaches traites I fois par jour le matin et suivies ensuite par leurs veaux jusqu'à 18 heures (tabl. 2). Dans ce dernier cas, les veaux tétaient leur mère durant la journée et étaient enfermés le soir vers $\mathrm{I} 8$ heures. Au début et à la fin des traites, les veaux tétaient un peu leur mère.

La méthode d'observation utilisée a fait l'objet d'une note technique (PETIT, I969).

L'activité des troupeaux était surtout diurne et étroitement liée aux heures du lever et du coucher du soleil (fig. I). En octobre cependant, on a enregistré vers minuit une activité importante du troupeau par nuit claire.

Les vaches allaitantes ont pâturé en moyenne durant 8 heures pendant la journée (du lever des premiers animaux Ie matin, au coucher des derniers le soir). Le temps de pâturage a été plus élevé en aô̂t qu'en juin-juillet (diminution de la qualité du pâturage) mais plus faible en octobre qu'en aon̂t (réduction du temps diurne) (tabl. 3). Pour un même mode de conduite (vaches allaitantes), le temps de pâturage a été plus élevé en juin-juillet ( + I 7 p. Ioo) sur les pâturages de moins bonne qualité. Pour des pâturages de qualité analogue, la réduction de 4 à 5 heures du temps disponible par la traite a diminué le temps de pâturage des vaches de I heure à $\mathrm{I}$ heure et demie. Lorsque les conditions météorologiques étaient mauvaises, les vaches s'arrêtaient de pâturer mais pâturaient ensuite avec plus d'intensité, soit le jour même, soit le lendemain (tabl. 4).

Le temps de pâturage journalier des veaux des vaches allaitantes a augmenté constamment avec l'âge (tabl. 3, fig. 3) ; il était beaucoup plus élevé (+ 29 p. Ioo) lorsque le pâturage était de mauvaise qualité. Les veaux des mères traites 2 fois par jour ont pâturé en moyenne pendant 33 minutes de plus que les veaux des vaches allaitantes sur des pâturages de qualité analogue, ceux des vaches traites a fois par jour, enfermés le soir, 55 minutes de moins. Ceci peut expliquer la croissance relativement faible des veaux des vaches traites $\mathbf{I}$ fois par jour (tabl. 7). Les mauvaises conditions météorologiques ne semblent pas avoir eu d'influence sur le temps de pâturage des veaux (tabl. 4).

La réduction du temps disponible ou la diminution de la quantité d'herbe a diminué le nombre des repas et allongé leur durée (fig. 2 et 4 ). Le comportement des veaux au pâturage se rapprochait de celui de leur mère quand ils avançaient en âge.

Les veaux des vaches allaitantes ont tété environ durant 40 minutes par 24 heures en 3 ou 4 fois par jour (tabl. 5), surtout le matin, le soir, et au début des cycles de pâturage. Les veaux des vaches traites une fois par jour ont tété durant I 5 à 20 minutes pendant qu'ils restaient en liberté avec leur mère. 
Les troupeaux effectuaient, durant la journée, des circuits réguliers sur les pâturages non cloisonnés et se couchaient toujours aux mêmes endroits la nuit. La dispersion des troupeaux était plus importante en automne qu'au printenips. Les animaux ont surpâturé les zones fertilisées et délaissé les zones pauvres (tabl. 6). Le temps de pâturage dans les marécages était cependant voisin de I heure et demic en automne.

Les résultats sont comparés à ceux obtenus par d'autres auteurs et on a essayé d'interpréter, à partir du comportement au pâturage, les différences de croissance des veaux selon le mode de conduite du troupeau.

\section{INTRODUCTION}

Dans le cadre d'une recherche coopérative sur la région de l'Aubrac (Ig64-Ig66). nous avons commencé l'étude de l'utilisation des pâturages d'altitude par des trou peaux de vaches allaitant leurs veaux, en observant le comportement de ces trou peaux.

L'Aubrac, situé au sud-ouest du Massif central, est un plateau en majeure partie basaltique, au climat rude, venteux et pluvieux (I ooo à I $200 \mathrm{~mm} / \mathrm{an}$ ). Il comporte, entre I ooo et I 300 mètres d'altitude, environ 300 pâturages ou "montagnes", de surface importante ( 40 à I Io ha) et généralement d'un seul tenant. Des troupeaux de vaches de race $A u b r a c$, d'effectifs importants (25 à roo environ), y estivent avec leurs veaux du début mai au milieu octobre (dates traditionnelles : 25 mai et 13 octobre). La race $A$ ubrac est une race rustique de petit format (VISSAC, r970). Les vaches (500 $\mathrm{kg}$ de poids vif environ) sont très fécondes et vêlent avec facilité ; les vêlages ont lieu à l'étable au début de l'année (janvier, février, mars).

Traditionnellement, les vaches étaient traites deux fois par jour à l'étable puis au pâturage près d'un petit enclos ou parc de traite ; le lait était transformé sur place en fromage dans le "buron ", petite construction de pierre tenant lieu d'habitation et de fromagerie. La faible production laitière (de I 500 à 22001 par lactation) et les conditions de vie très rudes en altitude ont conduit à l'abandon progressif de la traite et à l'apparition de nouveaux modes de conduite : I traite par jour plus allaitement du veau, allaitement du veau et zéro traite. En I964, sur 303 " montagnes 》 recensées les vaches n'étaient traites que sur 6I d'entre elles, soit 2 fois par jour le matin et l'après-midi (52 "montagnes "), soit une fois par jour le matin (9 "montaģnes )). Dans les autres " montagnes ", les vaches allaitaient leurs veaux en liberté avec elles ; dans la plupart des cas, les veaux étaient alors croisés Charolais.

La qualité des pâturages varie avec la quantité d'herbe disponible et la nature de la flore, qui sont surtout liées au bioclimat et à l'hétérogénéité de la couverture végétale (plus ou moins de rocailles et de marécages), et au mode d'exploitation du pâturage (cloisonnement ou pas, fertilisation par le déplacement du parc de traite). Enfin, les animaux disposent parfois d'un abri sommaire : bois ou bosquet, parc de traite ou murets.

A 1'aide d'un enregistrement visuel (PETIT, I969), nous avons décrit le comportement des troupeaux de vaches allaitantes sur les montagnes de l'Aubrac, en particulier leur emploi du temps et leur déplacement sur le pâturage. Nous avons ensuite analysé l'influence des différents modes de conduite et de la qualité des montagnes sur le comportement des animaux, et tenté d'interpréter les différences enregistrées dans la croissance des veaux. 
L'exploitation du pâturage par des troupeaux de vaches allaitantes a fait l'objet d'un certain nombre d'études :

Johnstone-Wallace et Kennedy (I944), Peiterson et Woolfolik (I955), Wagnon (I963) aux U. S. A., Walker (Ig62) en Nouvelle-Zélande, Chambers (I959) en Grande-Bretagne, Hutchison et al. (I962) au Tanganyika. Toutefois, elles ne comportent généralement pas l'enregistrement simultané du pâturage des vaches, des tétées et du pâturage des veaux sur des effectifs importants.

\section{MATÉRIEL, ET MÉTHODES}

\section{Choix des montagnes}

BÉRANGer et Tessier (I970) avaient mesuré lc gain de poids en été de 607 veaux répartis sur 22 "montagnes", et analysé l'influence de certains facteurs : mode de conduite du troupeau, race, sexe, qualité du pâturage ; les veaux étaient pesés à la montée et à la descente de la "montagne " et les quantités de lait éventuellement traites étaient mesurées.

Nous avons choisi 6 de ces "montagnes", d'une superficie de 43 à 90 ha, généralement d'un seul tenant, où estivaient des troupeaux de 29 à 82 vaches-mères (tabl. I) :

- quatre "montagnes" où les vaches n'étaicnt pas traites, dont deux de bonne qualité (Estremailles : $M_{1}$, Ruols : $M_{2}$ ) et deux de qualité moyenne ou médiocre (Plèches : $\mathbf{M}_{3}$, ChampSouveyrou : $\mathrm{M}_{4}$ ) ;

- deux "montagnes " où les vaches étaient traites, toutes deux de qualité moyenne. Sur l'une (Canuc: $\mathrm{T}_{1}$ ), elles étaient traites une fois par jour, le matin à l'aube ; sur l'autre (PuechCremat : ' $T_{2}$ ) deux fois par jour, le matin et l'après-midi à partir de ${ }_{5} \mathrm{~h}$ 3o. Sur $T_{1}$, les veaux restaient cn liberté avec leurs mères durant la journée et, durant la soirée et la nuit, étaient cnfermés dans le parc de traite. Sur $T_{2}$, ils étaient séparés de leurs mères toute la journée et pâturaient une parcelle close attenante au parc où ils rentraient d'eux-mêmes le soir. Dans les deux cas, la présence des veaux était indispensable au bon déroulement rle la traite ; ils intervenaient au début (amorçage) et à la fin (égouttage), ct restaicnt toujours présents, attachés près de leur mère. Une quantité limitée de foin leur était distribuée le soir dans le parc.

Les veaux étaient de race $A u b r a c$ sur les trois montagnes $\mathrm{T}_{1}, \mathrm{~T}_{2}$, et $\mathrm{M}_{4}, A u b r a c \times$ Charolais sur les trois autres $\mathrm{M}_{1}, \mathrm{M}_{2}$ et $\mathrm{M}_{3}$.

\section{Mesures}

Nous avons observé les troupeaux à 3 périodes (fin juin-début juillet, mi-août, début octobre) et, compte tenu du climat de la région, sous des conditions météorologiques variées. Chaque période comportait une journée d'observation des troupeaux de $T_{1}$ et $\mathrm{T}_{2}$, d'au moins un des troupcaux de $\mathrm{M}_{1}, \mathrm{M}_{2}, \mathrm{M}_{3}$ et $\mathrm{M}_{4}$.

L'enregistrement a porté sur l'activité globale cles troupeaux : pâturage des mères et des veaux, tétées, station debout et couchée, éventuellement déplacement des animaux sur la " montagne ". Nous considérions qu'un animal pâturait lorsqu'il avait la tête au niveau de l'herbe, ce qui correspondait au choix de l'herbe et à la préhension proprement dite. Nous n'avons pas enregistré la rumination; il nous était, en effet, difficile de l'observer sur un grand nombre d'animaux, et elle est essentiellement nocturne (HAxcock, I953).

Les observations avaient lieu à intervalle de 10 minutes, du lever des premiers animaux le matin, au coucher des derniers le soir, soit pendant $I_{7}$ heures en été et I 4 heures en automne (tabl. 2) ; nous avons aussi réalisé 5 enregistrements nocturnes. La méthode utilisée pour l'enregistrement de l'emploi du temps des animaux a fait l'objet d'une note (Petit, ig6g). Pour les 4 troupeaux de vaches non traites, nous avons noté la position des groupes d'animaux ou des individus isolés sur un plan ronéotypé des " montagnes ", à intervalle de Io minutes sur $M_{3}$, à intervalle de 20 minutes sur $\mathrm{M}_{1}, \mathrm{M}_{2}$ et $\mathrm{M}_{\mathbf{4}}$.

\section{Mode d'expression et d'interprétation des résultats}

En supposant constante l'activité du troupeau entre deux observations successives, nous avons calculé : 
M. PETIT

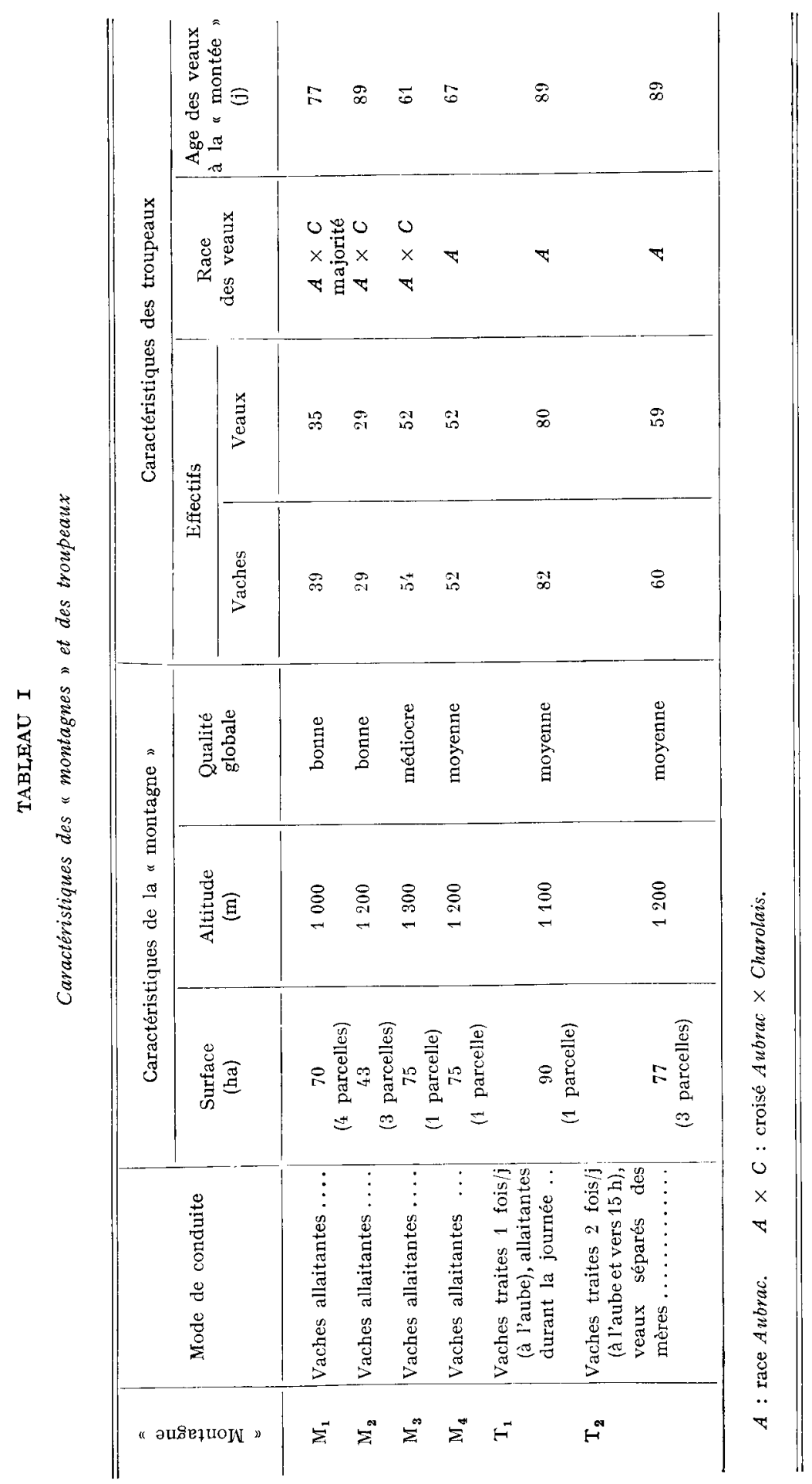


TABLEAU 2

Conditions d'enregistrement

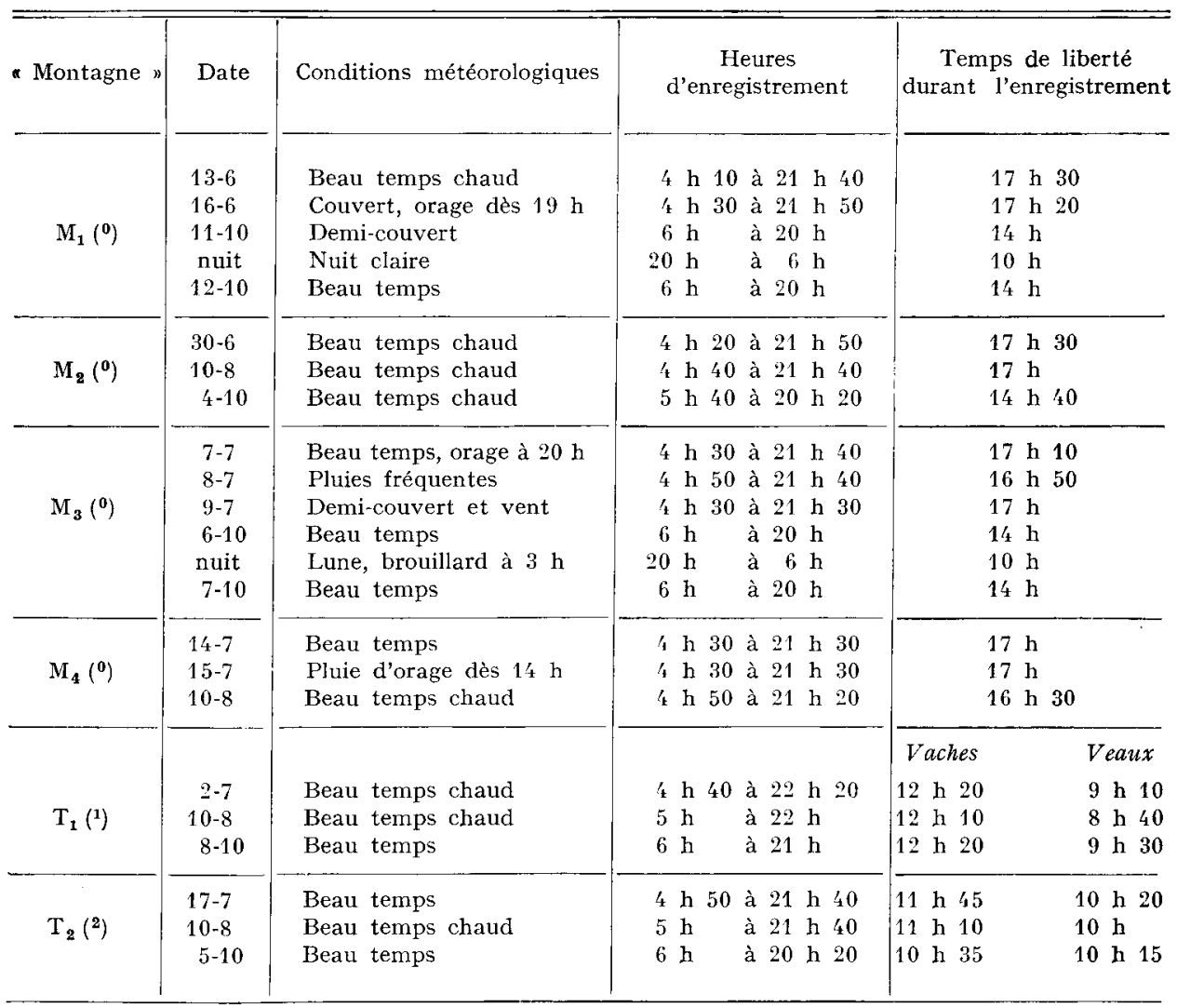

(0) : Vaches allaitant leur veau en totale liberté.

(1) : Vaches traites une fois/jour et veaux en liberté avec leur mère durant la journée.

Le 2-7: traite de $4 \mathrm{~h} 40$ à $8 \mathrm{~h} 50$, veaux parqués à $18 \mathrm{~h}$.

Le 10-8: traite de $5 \mathrm{~h}$ à $8 \mathrm{~h} 50$, veaux parqués à $17 \mathrm{~h} 30$.

Le 8-10: traite de $6 \mathrm{~h}$ à $8 \mathrm{~h}$, veaux parqués à $17 \mathrm{~h} 30$.

$\left.{ }^{2}\right)$ : Vaches traites deux fois/jour et veaux séparés de leur mère.

Le 17-7: traite de 4 h 50 à $7 \mathrm{~h} 20$ et de $15 \mathrm{~h}$ à $17 \mathrm{~h} 30$.

Le 10-8: traite de 5 h à $7 \mathrm{~h} 50$ et de 15 h 20 à $18 \mathrm{~h}$.

Le $5-10$ : traite de 6 h à 8 h 10 et de 15 h 30 à 16 h 50 .

- Le temps journalier consacré, en moyenne, par chaque animal au pâturage, à la tétée, aux stations debout et couchée :

$$
\text { Temps passé à une activité }=\mathrm{T} a=\frac{\sum(a i \times n)}{\mathrm{A}}
$$

où :

$a i=$ nombre d'animaux exerçant cette activité dans l'intervalle d'ordre $i$,

$A=$ nombre d'animaux du troupeau,

$n=$ durée de l'intervalle entre deux observations successives.

- La part relative ou fréquence de chaque activité du troupeau, que nous avions définie par l'expression. 
Dans l'intervalle d’ordre $i$, elle est égale à $\frac{a i}{\mathrm{~A}}$; pour la journée, elle est égale à $\frac{\sum a i}{\mathrm{NA}}=\frac{\mathrm{T} a}{\mathrm{~T}}, \mathrm{~N}$ étant le nombre d'observations journalières et $T$ le temps total d'observation.

Nous avons tracé, par jour et par troupeau, les histogrammes de fréquence du pâturage et de la tétée, en fonction de la période élémentaire de contrôle. Ces activités s'étant révélées cycliques, nous avons essayé de bien en distinguer les différents cycles. Les cycles de tétées du troupeau sont en général bien définis dans le temps. En revanche, le décalage horaire des cycles de pâturage individuels peut faire plus ou moins se confondre deux cycles de pâturage d'un troupeau; nous avons alors choisi le critère suivant : deux périodes voisines de pâturage présentant des effectifs maximum enregistrés $E_{1}$ et $E_{2}$, sont 2 cycles distincts de pâturage, si trente minutes au moins les séparent, pendant lesquelles on a enregistré moins de $\frac{E_{1}+E_{2}}{4}$ animaux qui pâturaient.

\section{RÉSULTATS}

Les activités des troupeaux de vaches allaitantes furent essentiellement diurnes. Comme la plupart des auteurs (revue bibliographique de HaNcock, I953), nous avons constaté qu'elles étaient étroitement liées aux heures de lever et de coucher du soleil (fig. I) : les premiers animaux se levaient dès les premières lueurs matinales, vers

30 Juin

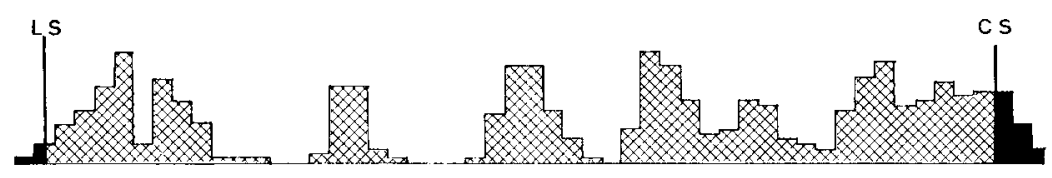

10 Août

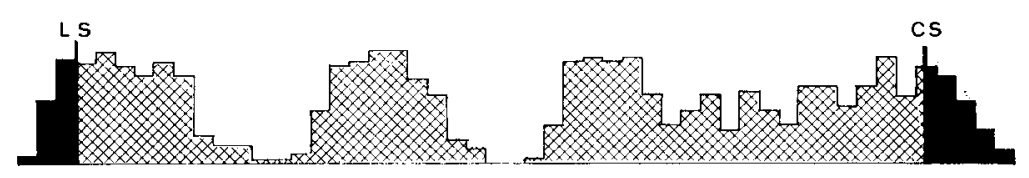

4 Octotre

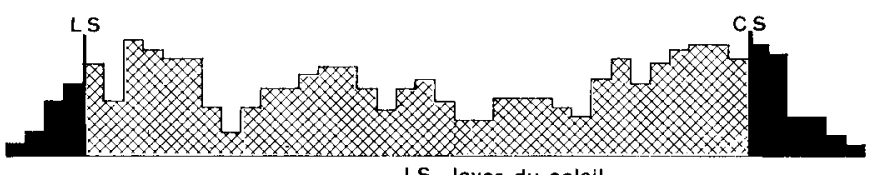

LS = lever du soleil.

CS = coucher du soleil.

$\begin{array}{lllllllllllllllllllll}4 & 5 & 6 & 7 & 8 & 9 & 10 & 11 & 12 & 13 & 14 & 15 & 16 & 17 & 18 & 19 & 20 & 21 & 22 \\ \text { heures }\end{array}$

FIG. I - Relation entre le temps diurne et l'activitê du troupeau

(pâturage sur la montagne M2)

$4 \mathrm{~h}$ I 5 en juin, $4 \mathrm{~h} 45$ en août et $6 \mathrm{~h}$ en octobre, soit respectivement $\mathrm{x} / 2 \mathrm{~h}, 3 / 4$ d'heure et $\mathrm{I} h$ environ avant l'heure du lever du soleil; les derniers se couchaient le soir vers $2 \mathrm{I} \mathrm{h} 30$ en juin, $2 \mathrm{I} h 20$ en août et $20 \mathrm{~h}$ en octobre, soit respectivement $\mathrm{I} / 2 \mathrm{~h}, \mathrm{I} \mathrm{h}$ et I $h$ 30 après l'heure du coucher du soleil. Nous appellerons " diurne " cette période comprise entre le lever et le coucher du troupeau.

La traite sur les "montagnes " $T_{1}$ et $T_{2}$ s'effectuait en présence des veaux et durait de 2 à 3 heures, selon l'effectif des troupeaux et le nombre de vachers ; dans ces deux systèmes, vaches et veaux ne disposaient donc pas librement de l'emploi de leur temps. 
L'emploi du temps diurne des troupeaux a été cyclique pour toutes les activités. Dans le cas des troupeaux de vaches allaitantes il est apparu réglé par les repas des vaches (fig. 2): un cycle de pâturage débutait souvent par un cycle de tétées (quand les vaches se levaient) et était toujours suivi par un cycle de repos (station debout ou couchée) incluant des périodes de rumination. Le premier cycle de pâturage et de tétée débutait le matin aussitôt après le lever du troupeau ; le dernier cycle de pâturage suivi du dernier cycle de tétée se terminait le soir juste avant le coucher des animaux.

\section{PÂTURAGE}

\section{Pâturage diurne des vaches}

\section{Temps de pâturage.}

Pour les 2r jours d'observation, le temps de pâturage des vaches a été compris entre 6 et 9 heures (tab1. 3), ce qui est en accord avec les résultats généralement obte-

TABLEAU 3

Temps de paturage des vaches et des veaux

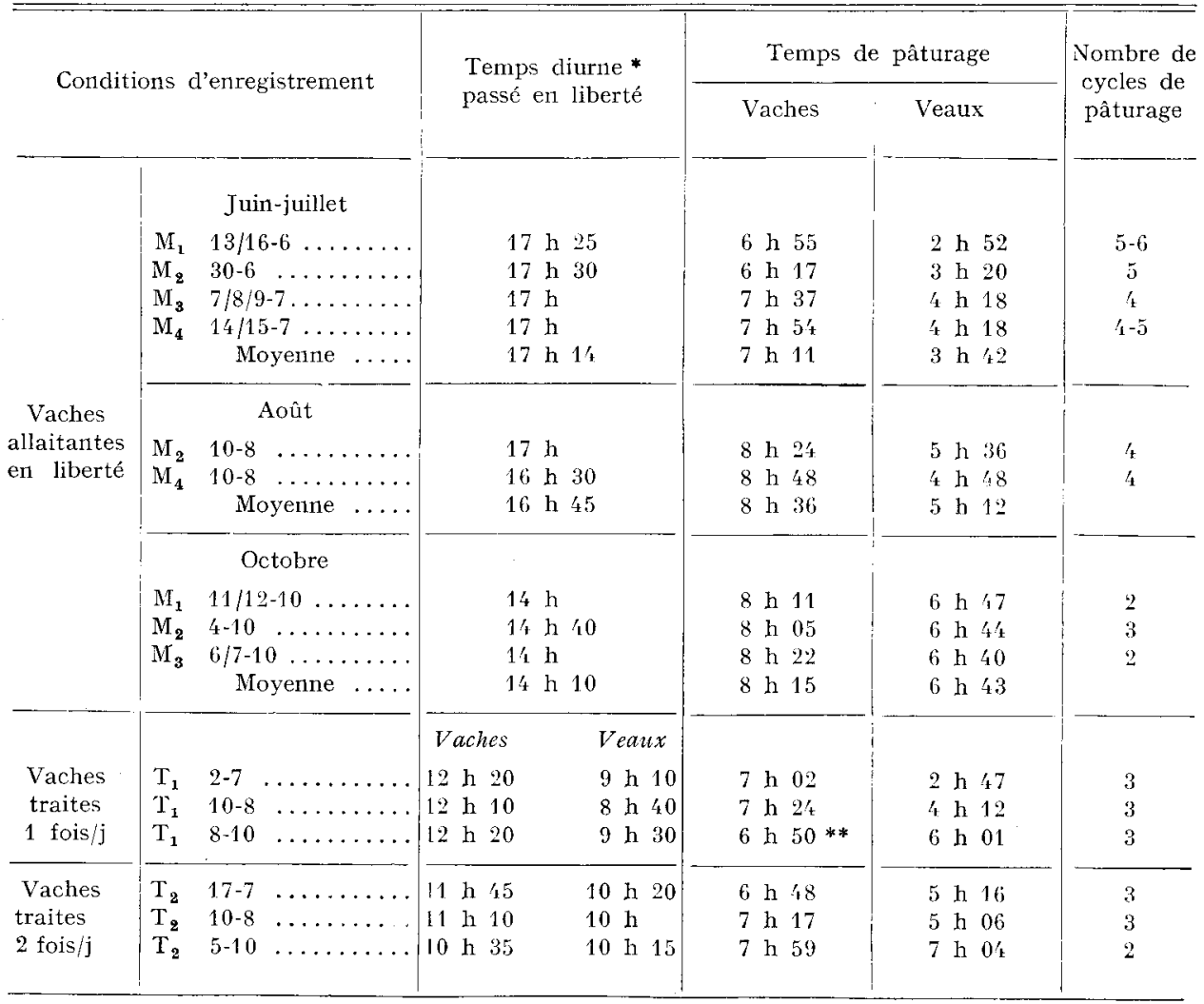

Nota : Les pâturages ont été regroupés selon leur qualité $=\mathrm{M}_{1}$ et $\mathrm{M}_{2}$ d'une part, $\mathrm{M}_{3}$ et $\mathrm{M}_{4}$ d'autre part.

* Temps diurne : entre le lever et le coucher du troupeau.

** Les vaches de $T_{1}$, pour une raison inconnue, ont très peu pâturé le soir du 8-10. 


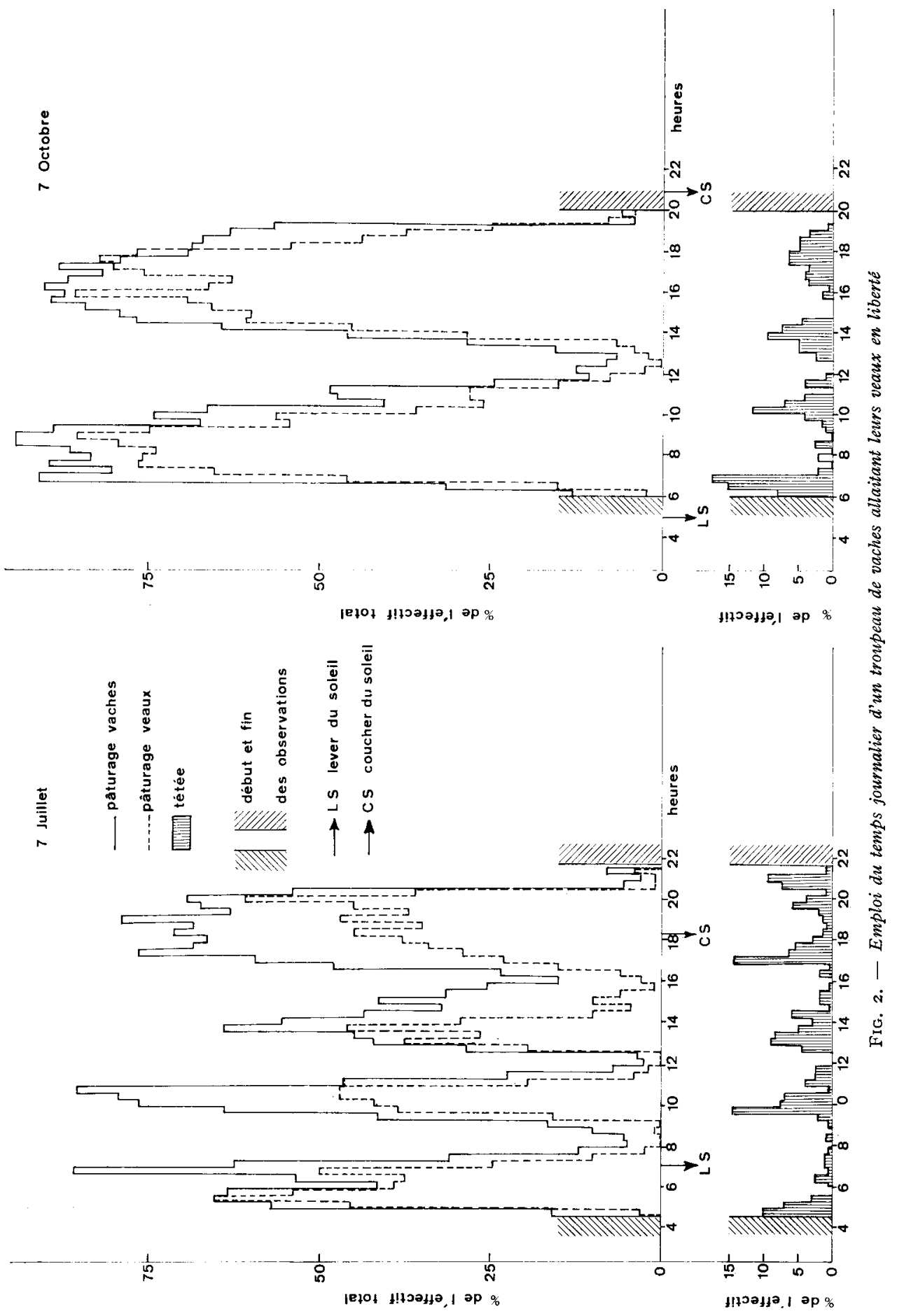


nus sur gros bovins. Il a varié surtout avec la durée du temps disponible (mode de conduite et saison), et la qualité du pâturage (saison et qualité des "montagnes").

L,e temps de pâturage diurne des vaches allaitantes a été en moyenne de $8 \mathrm{~h}$ or. Cependant, il a augmenté de 85 minutes de la fin juin à la mi-août et a diminué ensuite de 2 I minutes d'aồt à octobre. Exprimé en p. Ioo du temps diurne (entre le lever du troupeau le matin et le coucher des derniers animaux le soir), il a augmenté de 42 à 5 I puis à 58 .

Les vaches traites 2 fois par jour ont pâturé durant 7 h $2 I$ en moyenne; leur temps de pâturage a augmenté de 29 minutes de juillet à août, de 42 minutes d'août à octobre, représentant respectivement 58,65 et 75 p. Ioo du temps diurne disponible. Celles traites une fois par jour ont pâturé durant $7 \mathrm{~h} 02$ en juillet, $7 \mathrm{~h} 24$ en août; en octobre, le 8 , pour une raison inconnue, elles n'ont que très peu pâturé le soir après la rentrée des veaux au parc.

\section{- Iufuence du mode de conduite.}

Les vaches traites ont pâturé moins longtemps que les vaches allaitantes, surtout en juillet et août : (fig. 3, tabl. 3), 57 et 43 minutes en moins en juillet pour les vaches de $T_{2}$ et $T_{1}$ respectivement, I h $3 \mathrm{I}$ et $\mathrm{I}$ h 24 en août, par rapport aux vaches allaitantes pâturant des "montagnes » de qualité analogue $\left(M_{3}\right.$ et $\left.M_{1}\right)$. Cela est vraisemblablement dû aux différences de temps diurne disponible; en juillet-août la traite durait $5 \mathrm{~h}$ en ' $T_{2}$ et $4 \mathrm{~h}$ Io en 'T $T_{1}$. Les vaches n'ont pas pu compenser ce manque de temps libre par l'augmentation, pourtant importante, de la part de ce temps passée à pâturer. En automne, la diminution du temps de traite en $\mathrm{T}_{2}(3 \mathrm{~h} 30)$ a réduit la différence de temps de pâturage entre vache traite 2 fois par jour et vaches allaitantes (23 minutes).

\section{- Influence de la saison.}

Le temps de pâturage des vaches a augmenté du début à la fin de la saison pour les vaches traites, et du début au milieu de la saison pour les vaches allaitantes, malgré une légère réduction du temps disponible, (fig. 3). Nous pouvons, sans doute, attribuer cette augmentation à l'évolution de la qualité du pâturage, en particulier à une herbe moins abondante, plus rase et peut-être de moindre qualité. Cependant, de août à octobre, la forte diminution du temps disponible des vaches allaitantes a entraîné, malgré l'augmentation de la part de ce temps passée à pâturer, une diminution du cemps de pâturage diurne.

\section{- Infuence de la qualité des "montagnes".}

L'influence de la qualité du pâturage s'est aussi manifestée entre " montagnes ». L,e temps de pâturage est resté supérieur, tout au cours de la saison, sur les " montagnes » de moins bonne qualité, probablement parce que la quantité d'herbe disponible y était plus faible (tabl. 3). La différence qui atteignait + I7 p. Ioo en juin-juillet s'est progressivement atténuée pour n'être plus que de $3 \mathrm{p}$. Ioo en octobre; l'amplitude des variations journalières en automne était supérieure aux différences enregistrées entre les deux catégories de "montagnes ».

D'une façon générale, le temps de pâturage journalier des animaux a augmenté quand la qualité du pâturage a diminué, qu'il s'agisse de l'influence de la saison ou des différences entre " montagnes " à une même époque. JoHnSTONE-WALLACE et KEN- 
M. PETIT

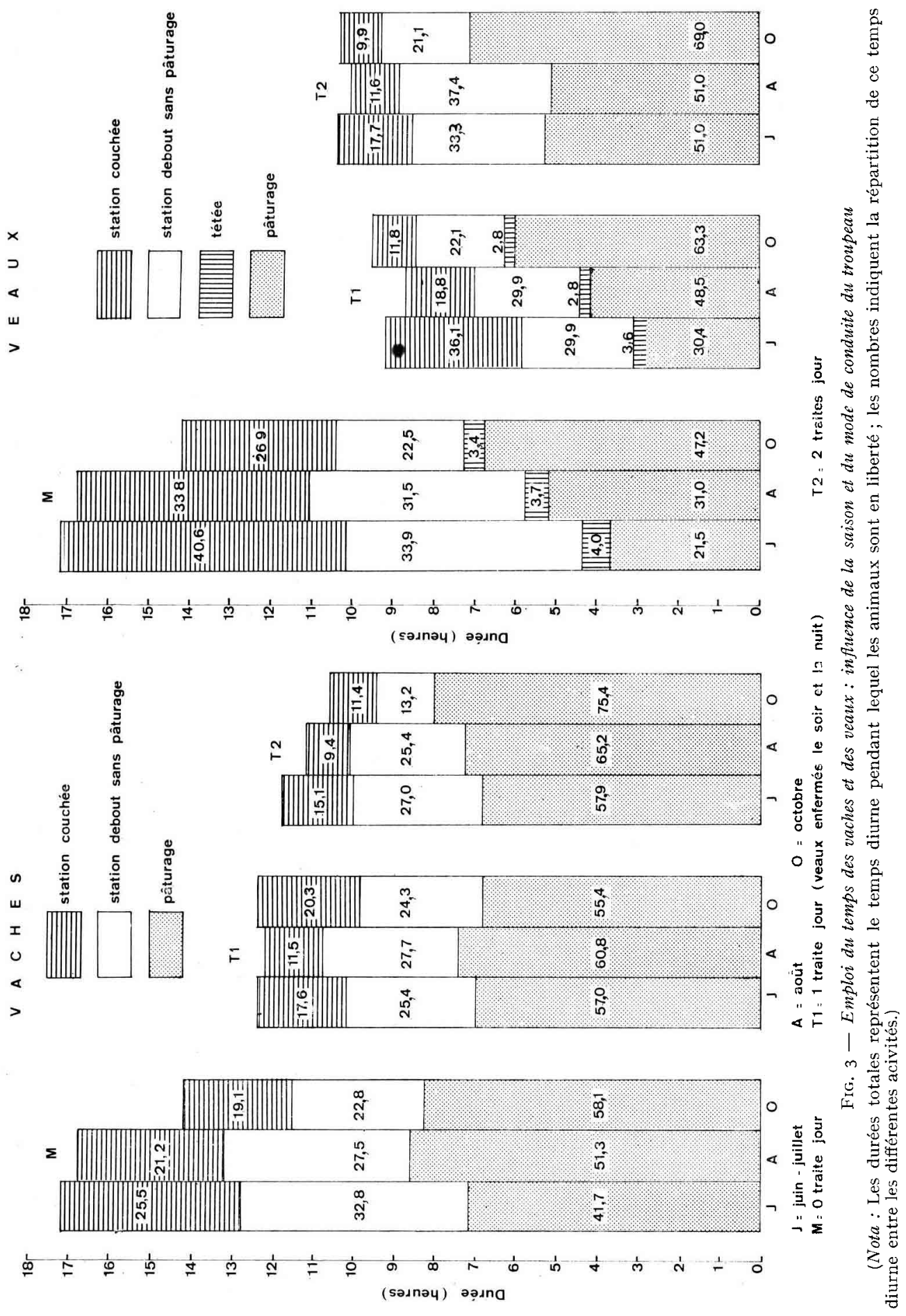


NEDY, aux U.S.A., trouvent des temps de pâturage à peu près constants ( 7 à $8 \mathrm{~h}$ ) pour des quantités d'herbe disponible allant de $25^{\circ}$ à $\mathbf{I}$ ooo livres/acre, mais HANCoCK (I953) constate que la majorité des auteurs s'accorde sur une augmentation du temps de pâturage (de plus d'une heure parfois) quand la quantité d'herbe diminue.

La diminution de la quantité d'herbe fut très apparente sur les " montagnes " étudiées, mais nous ne pouvions pas dissocier les deux facteurs, quantité et qualité de l'herbe disponible. Nous avons seulement constaté que les animaux effectuaient un tri plus important de l'herbe au milieu de la saison plutôt qu'au début, d'une part sur les "montagnes " de qualité médiocre, d'autre part sur celles de bonne qualité. I a raréfaction et la mauvaise qualité de 1'herbe sur tous les pâturages en automne, ont sans doute réduit les différences de temps de pâturage enregistrées selon la qualité des « montagnes ».

\section{- Influence des conditions météorologiques.}

Dans de bonnes conditions météorologiques, stables, les séries d'observations sur une même "montagne " $\left(M_{1}\right.$ : I3 et I6 juin, $M_{3}: 6$ et 7 octobre, $M_{1}$ : II et I2 octobre) n'ont pas montré de fluctuations sensibles de l'emploi du temps des vaches. En revanche, deux séries d'observations du début de la saison $\left(\mathrm{M}_{3}: 7,8\right.$ et 9 juillet, $M_{1}$ : I4 et $I_{5}$ juillet) ont mis en évidence des variations journalières du temps de pâturage quand les conditions météorologiques n'étaient pas stables (tabl. 4). Le temps de pâturage a été plus faible de 30 minutes en $\mathrm{M}_{1}$ lors d'une journée pluvieuse suivant une journée de beau temps. Le lendemain d'une journée pluvieuse en $\mathrm{M}_{3}$, il a augmenté de $5^{8}$ minutes. HANCock (I953) signale qu'il est assez rare d'observer une réduction importante du temps de pâturage diurne par temps pluvieux et que les animaux pâturent plus longtemps les jours suivants.

\section{Répartition journalière.}

\section{- Vaches allaitantes.}

A chaque cycle de pâturage du troupeau correspondait un cycle de pâturage individuel. Le nombre de cycles de pâturage des vaches allaitantes a diminué à mesure que la saison avançait, passant de 4 ou 5 à 2 ou $3\left(M_{2}: 5\right.$ en juin, 4 en août et 3 en octobre). Cela semble résulter d'une fusion des cycles de l'après-midi, puis d'une nouvelle fusion des cycles du matin, pouvant supprimer totalement les cycles de pâturage intermédiaires. De plus, le pourcentage maximum d'animaux pâturant au milieu des cycles a augmenté, de 84 en juin-juillet à 96 en octobre. Le temps de pâturage par cycle est ainsi passé de I-2 heures à 4 heures environ. Au début de la saison, sur les " montagnes " de qualité médiocre, le nombre de cycles de pâturage était inférieur et leur durée supérieure à ceux enregistrés sur les " montagnes » de bonne qualité : 5 à 6 cycles en $M_{1}, 5$ en $M_{2}, 4$ à 5 en $M_{4}$ et 4 en $M_{3}$. Warker (I962), en NouvelleZélande, n'a pas noté de diminution du nombre des cycles de pâturage au cours de la saison ( 5 à 6 cycles de novembre à février), mais ses enregistrements furent toujours réalisés le lendemain d'un changement de parcelle. La majorité des auteurs (HANCOCK, I953) s'accorde sur une diminution du nombre de cycles quand la quantité d'herbe diminue.

Les deux cycles du matin et du soir étaient toujours les deux plus importants de la journée. Pour I4 jours d'observations sur I5, celui du soir a été le plus long : 


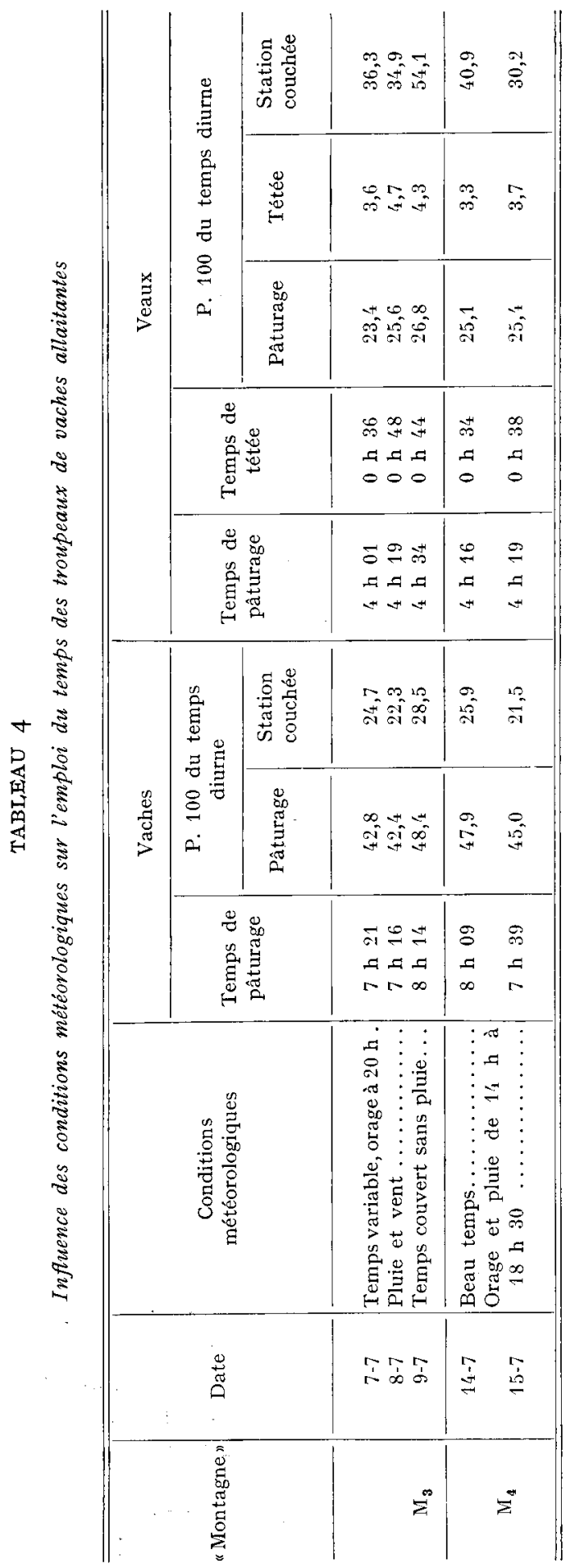


32,6 (26 à 40) p. Ioo du temps de pâturage journalier au printemps, 55,6 (45 à 63) p. Ioo en automne. Le milieu de la journée a toujours été marqué par une période de repos importante, entre II et I4 heures (fig. 2 ).

\section{- Vaches traites.}

Les vaches traites attendaient le vacher le matin souvent couchées. Elles se mettaient à pâturer aussitôt la fin de la traite vers 8 heures et s'arrêtaient au milieu de la journée (sauf en ' $\mathrm{T}_{2}$ en octobre). Elles reprenaient le pâturage jusqu'à la traite du soir (en ' $\mathrm{T}_{2}$ vers $\mathrm{I}_{5} \mathrm{~h} 3 \mathrm{j}$ ), ou jusqu'à une nouvelle période de repos coïncidant souvent avec la rentrée des veaux au parc en $T_{1}$. Débutait ensuite le cycle de pâturage du soir, jusqu'à la nuit (fig. 4). Les cycles de pâturage survenaient donc à des heures essentiellement dépendantes de la traite. Au début de la saison, leur nombre était de 3, au lieu de 4 pour les troupeaux de vaches allaitantes de $\mathrm{M}_{3}$ ou $\mathrm{M}_{1}$, "montagnes » de qualité voisine. A la fin de la saison, il était de 3 en $T_{1}$, de 2 en $T_{2}$ (tab1. 3 ). Les fréquences maximales de pâturage enregistrées au cours des cycles étaient toujours comprises entre go et IOO p. Ioo et supérieures à celles des vaches allaitantes ; nous retrouvons ici le fait que les vaches traites essayaient de compenser la réduction du temps disponible par un pâturage plus intense.

Lors de journées pluvieuses, les cycles de pâturage étaient hachés par les averses, mais reprenaient aussitôt avec des fréquences élevées; lorsque la pluie persistait, les animaux montraient une certaine accoutumance et le pâturage n'était interrompu que lorsqu'elle redoublait d'intensité. Il existe donc une certaine compensation possible au cours de la journée, en plus de celle des jours suivants. Nous avons constaté qu'une faible pluie, obligeant les animaux à se lever, pouvait être le départ d'un cycle de pâturage (en $\mathrm{M}_{4}$ le $\mathrm{I}_{4}$ juillet). Il ne nous est pas apparu de modifications sensibles de l'emploi du temps des animaux lors des quelques journées de chaleur relative, à l'occasion desquelles nous avons effectué des enregistrements au début de la saison. La majorité des auteurs s'accorde sur une modification de la répartition du pâturage en pays chauds (HaNCOCK, I953) ; les périodes de pâturage sont décalées aux moments de la journée où la température est plus frâiche, en particulier tôt le matin.

\section{Pâturage diurne des veaux.}

Le temps de pâturage des veaux des vaches allaitantes a toujours été inférieur à celui de leurs mères (tabl. 3, fig. 3) : il a augmenté considérablement tout au cours de la saison, de 3 h 42 à 6 h 43 ; rapporté au temps diurne, il est passé de 22 à 47 p. Ioo. Cela est dû̀, d'une part, à l'avancement en âge du veau et à la diminution de la production laitière de la mère, d'autre part, à la diminution de la qualité du pâturage. L'évolution du rapport : $\frac{\text { temps de pâturage des veaux }}{\text { temps de pâturage des vaches }}$ rend compte des deux premiers facteurs : il a augmenté de 52 à $8 \mathrm{r}$ p. roo. Cela confirme les résultats obtenus par Chambers (I959) et HuTCHison et al. (I962) qui notent une augmentation du temps de pâturage des veaux avec l'âge.

Suivant leurs mères au cours des déplacements, les veaux ont en général effectué le même nombre de cycles qu'elles. Ces cycles étaient bien distincts surtout le matin et le soir, même quand les veaux n'avaient que 3 à 4 mois. Aut début de la saison cependant, les fréquences maximales au milieu des cycles de pâturage n'étaient que 


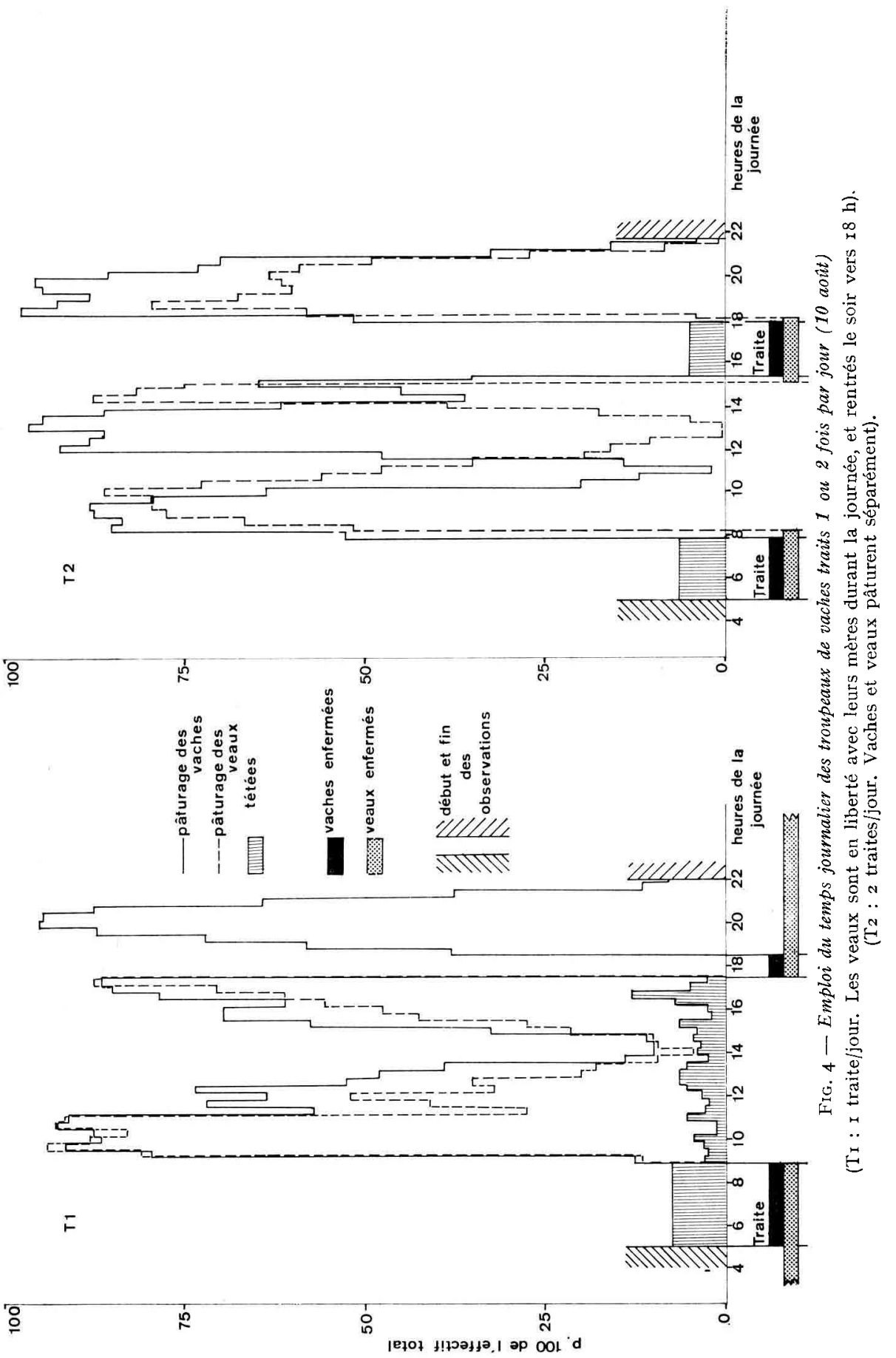


de 58 p. Ioo au lieu de $92 \mathrm{p}$. Ioo en automne, et les veaux terminaient souvent leurs repas plus tôt que leurs mères, comme s'ils étaient plus vite rassasiés. C'est peutêtre pourquoi nous avons observé, comme HuTchrson et al. (Ig62), qu'ils pouvaient parfois effectuer un cycle de plus qu'elles. Selon MAC AR'THUR (cité par Hancock), les veaux pâturent moins vite quand ils sont jeunes et sélectionnent davantage leur nourriture. D'une manière générale, le pâturage des veaux que nous avons observés se rapprochait d'autant plus de celui de leurs mères qu'ils avançaient en âge. Le cycle du matin était fréquemment le plus important pour les veaux au début de la saison ; en fin de saison, c'était le cycle du soir, comme pour les vaches.

Les veaux des vaches traites 2 fois par jour ont pâturé en moyenne durant 5 h 49 , toujours pendant plus de $5^{\circ} \mathrm{p}$. Ioo du temps durant lequel ils étaient en liberté. Cela représentait $5^{8}$ minutes en juillet de plus que les veaux des vaches allaitantes de " montagnes" de qualité analogue, et 24 minutes en octobre. Cette différence de temps de pâturage était vraisemblablement due aux différences de quantités de lait bues ; elle a été surtout importante au début de la saison quand le lait bu représentait l'essentiel de la ration des veaux des vaches allaitantes. Il est à noter, cependant, que ceux-ci étaient plus jeunes de 25 jours. Les veaux de $\mathrm{T}_{\mathrm{z}}$ ont effectué le même nombre de cycles de pâturage que leurs mères dont ils étaient séparés ; mais, bien que leurs parcelles respectives fussent contiguës, leurs heures de repas ne coïncidaient pas comme dans le cas de troupeaux de vaches allaitantes (fig. 4).

Les veaux des vaches traites une fois par jour étaient libres avec leurs mères durant $9 \mathrm{~h}$ environ. Ils ont pâturé durant $4 \mathrm{~h} 20$ en moyenne, soit 55 minutes de moins que les veaux des vaches allaitantes de "montagnes " analogues et $\mathrm{I} h 29 \mathrm{de}$ moins que les veaux des vaches traites deux fois par jour. Ces veaux, qui buvaient plus de lait que les veaux de $T_{2}$ et moins que ceux des vaches allaitantes, n'ont pas eu un temps de pâturage intermédiaire. Cette anomalie ne peut s'expliquer totalement par la réduction du temps diurne disponible inférieur de I heure seulement à celui des veaux de $\mathrm{T}_{2}$. Durant la journée, les veaux de $\mathrm{T}_{1}$ suivaient les vaches au cours des déplacements (fig. 4), au lieu de pâturer indépendamment aux heures de liberté comme ceux de $T_{2}$; ce phénomène a été très net quand les veaux étaient jeunes ( $2 \mathrm{~h} 47$ seulement de pâturage le 2 juillet). De plus, la rentrée au parc interrompait leur cycle de pâturage du soir, qui était très important pour leurs mères et pour les autres troupeaux (fig. 4). Ces trois raisons peuvent expliquer en partie le fait qu'ils aient pâturé moins longtemps que tous les autres veaux, en particulier ceux des vaches allaitantes qui disposaient pourtant de plus de lait. Après leur dernier cycle de pâturage, les veaux des deux " montagnes " $T_{1}$ et $T_{2}$ ont consommé en moyenne $400 \mathrm{~g}$ de foin à l'intérieur du parc.

Comme leurs mères, les veaux pâturent plus longtemps sur les "montagnes » de qualité médiocre : +29 p. roo au printemps en $\mathrm{M}_{3}$ et $\mathrm{M}_{1}$, " montagnes " de qualité médiocre, par rapport à $\mathbf{M}_{2}$, " montagne " de bonne qualité où les veaux étaient d'un âge comparable. Enfin, lors des journées pluvieuses, le temps de pâturage des veaux n'a pas diminué ; il a même augmenté de quelques minutes, peut-être parce que leur activité normalement réduite était stimulée par la pluie qui les obligeait à se lever. Le lendemain d'une journée pluvieuse en $\mathrm{M}_{3}$, le temps de pâturage des veaux a encore augmenté de $I_{5}$ minutes, probablement parce qu'ils suivaient leurs mères. 


\section{Pâturage nocturne}

Nous avons observé le troupeau de vaches allaitantes vers o et 2 heures lors de trois nuits et sur 3 " montagnes " différentes à la fin juin, début juillet; l'activité nocturne des troupeaux était alors réduite, mise à part la rumination : les animaux se levaient de temps en temps, mais ne pâturaient pas. En revanche, nous avons constaté une certaine activité nocturne en automne ; aussi avons-nous réalisé 2 enregistrements entre $20 \mathrm{~h}$ et $6 \mathrm{~h}$ sur les " montagnes " $\mathrm{M}_{1}$ et $\mathrm{M}_{3}$. Par pleine lune, sur la " montagne" $M_{1}$ (II-I2 octobre), le troupeau a effectué un cycle de pâturage entre $22 \mathrm{~h} 30$ et I h 30 , représentant $9 \mathrm{p}$. Ioo pour les veaux et I4 p. Ioo pour les vaches du temps de pâturage par 24 heures. Sur la "montagne " $\mathrm{M}_{3}$ (6-7 octobre), par nuit relativement claire, mais avec des nappes de brouillard, les vaches et les veaux n'ont que très peu pâturé (respectivement 3 et 2 p. roo) et de façon discontinue ; il est possible que le brouillard, très fréquent à cette époque sur les "montagnes ", gêne le pâturage nocturne des animaux.

WALKER (I962), en Nouvelle-Zélande, a observé vers minuit, de novembre à février, un pâturage de vaches allaitantes, mais dans cette étude, la nuit était plus longue qu'en Aubrac ( 2 heures environ de différence au début de l'été). WaGNON (I963) trouve un léger pâturage nocturne au cours des nuits de 7 à 8 heures dans un ranch californien en plein été. Le pâturage nocturne est parfois important en pays de climat chatd (Hutchison et al., I962).

C'est en octobre, à la période où la quantité d'herbe est la plus faible et le temps diurne disponible le plus réduit, que nous avons enregistré un pâturage nocturne. Grâce à ce pâturage nocturne, les vaches de $\mathrm{M}_{1}$ ont pâturé durant $9 \mathrm{~h} 30$ par 24 heures, donc plus longtemps qu'en août. C'est aussi à cette époque de l'année que l'activité " diurne " débordait le plus le temps diurne réel (fig. I). Nous n'avons malheureusement pas réalisé d'observations nocturnes sur les troupeaux de vaches traites.

\section{Autres activités}

\section{I. - Les tétées}

Lors des trois séries d'observations, les temps de tétées diurnes des veaux des vaches allaitantes qui avaient I IO, I55 et 2 I $_{4}$ jours d'âge en moyenne, ont été respectivement de 4 r, $3^{8}$ et 29 minutes (tabl. 5).

TABLEAU 5

Évolution du nombre et de la durée des tétées des veaux en liberté avec leurs mères

\begin{tabular}{|c|c|c|c|}
\hline Époque de l'annce & $\begin{array}{l}\text { Fin juin } \\
\text { début } \\
\text { juillet }\end{array}$ & Mii-août & $\begin{array}{l}\text { Début } \\
\text { octobre }\end{array}$ \\
\hline $\begin{array}{l}\text { Age moyen des veaux }(\mathrm{j}) \ldots \ldots \ldots \ldots \\
\text { Temps de tétécs journalier }(\mathrm{m} n) \ldots \\
\text { Nombre de tétées journalières } \ldots \ldots \ldots \\
\text { Durée d'une tétce }(\mathrm{mn}) \ldots \ldots \ldots \ldots \ldots\end{array}$ & 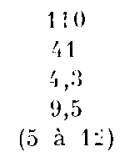 & $\begin{array}{c}155 \\
38 \\
3,2 \\
11 \\
(8 \text { à } 15)\end{array}$ & $\begin{array}{c}211 \\
29 \\
2,0 \\
15 \\
(10 \text { à }: 0)\end{array}$ \\
\hline
\end{tabular}


Les veaux des vaches traites une fois par jour ont tété durant I5 à 20 minutes (soit environ deux fois moins longtemps que ceux des vaches allaitantes) auxquelles il faut ajouter 16 minutes de tétée durant la traite du matin à laquelle le veau participe (amorçage et égouttage). Les veaux des vaches traites deux fois par jour ont tété lors des traites durant I3 minutes de juillet à août, durant 7 minutes en octobre (dont un amorçage de une minute à chaque traite).

Dans les troupeaux de vaches allaitantes, les tétées avaient lieu généralement au début des cycles de pâturage, sauf celles du soir qui se plaçaient juste avant le coucher du troupeau. Les tétées nocturnes étaient rares en été, mais ont représenté I9 p. Ioo du temps de tétées par 24 heures en octobre. Lors des deux enregistrements réalisés en octobre, les temps de tétées par 24 heures ont été de $3^{8}$ et 34 minutes pour des veaux âgés de r9o et 225 jours. Nous pouvons penser que les temps de tétées des veaux observés étaient voisins de 40 minutes environ par 24 heures et pour toute la saison, comme ceux trouvés par JOHNSTONE-WALLACE et KENNEDY (I944). HuTCHIson et al. (I962), au Tanganyika, ont obtenu 44 à $6 \mathrm{r}$ minutes de tétée par 24 heures dont la moitié environ durant la nuit (ro-r2 heures).

Les contrôles laitiers réalisés sur des vaches $A$ ubrac traites ont montré que leur production laitière en " montagne " était voisine de 6 à $7 \mathrm{~kg}$ en juin-juillet-août, mais diminuait ensuite rapidement jusqu'à $3 \mathrm{~kg}$ environ au début du mois d'octobre. S'il en est ainsi pour les vaches allaitantes, la vitesse d'ingestion moyenne au cours des tétées aurait alors été la plus faible en automne (les veaux prolongeaient d'ailleurs beaucoup leur succion et changeaient souvent de trayons). WAL,KER (I962) estime qu'il n'y a pas de relation évidente entre le temps de tétées et la production laitière des mères; aussi enregistre-t-il plutôt le nombre de tétées journalières.

Tous les veaux ne buvaient pas à chaque cycle de tétée du troupeau et nous ne pouvions déduire le nombre moyen de tétées individuelles du nombre de cycles de tétée; aussi avons nous mesuré la durée d'une dizaine de tétées lors de chaque jour d'observation et calculé le nombre de tétées en divisant le temps de tétée journalier par la durée moyenne d'une tétée. Nous avons obtenu 4 à 5 tétées par jour à 3,5 mois d'âge et 2 à 3 à 7 mois; la durée moyenne d'une tétée passait de Io à 15 minutes (tabl. 5). WALKER trouve, pour des veaux Hereford et Aberdeen $\times$ Angus âgés de 3,5 à 5,5 mois, de 2 à 6 tétées par 24 heures selon les individus. Chambers (I959) trouve 3 tétées diurnes de 8 à 16 minutes sur veaux $H$ ereford de o à 3 mois. Avec des zébus, Hu'TCHISON et al. (Ig62) obtiennent un nombre et un temps de tétées supérieurs mais décroissants de 3 à 6 mois (de 61 minutes en 7 tétées à 44-52 minutes en 5-6 tétées).

Les variations journalières du temps total de tétées, sur une même " montagne " à une même époque, semblaient essentiellement dues aux conditions météorologiques ; les temps de tétées étaient généralement accrus lors d'une journée pluvieuse (tab1. 4). De nombreuses tétées survenaient à la suite des averses, ce qui perturbait le rythme naturel des tétées.

\section{2. - Périodes de repos}

Dans les troupeaux de vaches allaitantes, vaches et veaux se reposaient durant I à 2 heures entre chaque cycle de pâturage, en restant alternativement debout ou couchés. Le nombre de périodes de repos a diminué de 3-4 à I-2 au cours de la saison. 
Celle du milieu de la journée était la plus importante ; elle durait de I h à I h 30 au printemps et 3 heures environ en automne.

Nous avons enregistré de façon précise le temps passé en station couchée. I1 représentait pour les vaches allaitantes un peu moins de la moitié du temps passé sans pâturage et pour leurs veaux un peu plus (fig. 3) ; ceux-ci restaient couchés deux fois plus longtemps que leurs mères au début de la saison et une fois et demie plus en octobre. Par temps de pluie, les animaux restaient couchés moins longtemps, surtout les veaux.

Dans les troupeaux de vaches traites, les animaux se reposaient entre les cycles de pâturage et lors de la traite ; les vaches et les veaux restaient couchés peu de temps durant la journée en dehors des périodes de traite (fig. 3).

\section{DÉPLACEMENT DES TROUPEAUX}

Nous avons observé les déplacements des vaches allaitantes et de leurs veaux sur les “montagnes " $\mathrm{M}_{1}, \mathrm{M}_{2}, \mathrm{M}_{3}$ et $\mathrm{M}_{1}$. Sur $\mathrm{M}_{3}$, nous avons noté la répartition des animaux sur les différentes zones de végétation.

\section{I. - Déplacement et groupement des animaux}

Les déplacements des troupeaux que nous avons observés étaient collectifs et guidés par les vaches ; ils avaient lieu lors des cycles de pâturage. D’autres déplacements individuels pouvaient survenir au cours des périodes de repos, les animaux flânant.

Le matin, les troupeaux quittaient leur aire de couchage nocturne; elle était très localisée au printemps et en été, proche du buron (habitation des vachers et fromagerie) pour les quatre "montagnes "; en automne, les animaux passaient la nuit en petits groupes plus dispersés mais dans la même zone, proche de celle du printemps. La surface couverte durant la nuit par le troupeau de $\mathbf{M}_{3}$ (polygone convexe enveloppant les animaux) a été de $I, 2$ ha au printemps et 9,o ha en automne.

Sur les deux " montagnes " d'un seul tenant, $M_{3}$ et $M_{1}$, les troupeaux effectuaient au cours de la journée un circuit, en tournant dans un certain sens qui est resté le même tout au long de la saison. Ce circuit est à rapprocher de celui qu'effectuaient les animaux lors de l'ancienne pratique des "ayguades » : traditionnellement le pâtre faisait effectuer aux vaches le tour de la "montagne " durant la journée et les laissait au centre durant la nuit ; le fait que les animaux aient passé très souvent la nuit près du buron peut d'ailleurs s'expliquer de façon analogue. Sur les " montagnes » $M_{1}$ et $M_{2}$, qui étaient cloisonnées en plusieurs parcelles, nous n'avons pas observé la même régularité dans le parcours des animaux.

L,e circuit journalier des animaux nous a paru être influencé par les conditions météorologiques; c'est ainsi qu'en $\mathrm{M}_{4}$, le I5 juillet, le troupeau est revenu trois fois au même endroit, en lisière d'un bois, entre I4 et 18 heures, pour s'abriter des rafales de pluie. En $\mathrm{M}_{1}$, le $\mathrm{I}_{3}$ juin, journée relativement chaude, les déplacements journaliers du troupeau s'effectuaient en étoile autour du seul point d'eau de la parcelle.

Les déplacements étaient collectifs, les animaux restant plus ou moins groupés selon leurs activités et l'époque de la saison. Lors des périodes de repos diurne ils étaient généralement bien groupés, sur le haut des buttes herbeuses ou pierreuses, surtout au début de la saison ou par temps chaud. Ia surface moyenne occupée par le 
troupeau de $\mathrm{M}_{3}$, entre les cycles de pâturage quand l'effectif d'animaux au repos était maximal, fut de 2,6 ha au printemps et de 3,9 ha en automne. Lors des cycles de pâturage, le troupeau se déployait ; les animaux étaient plus dispersés en octobre qu'en juin-juillet. En $\mathrm{M}_{3}$, la surface moyenne couverte par le troupeau fut, au milieu des cycles de pâturage journaliers, de 4,o ha en juillet (I 2 observations) et de Io,o ha en octobre (4 observations) ; la plus faible quantité et la moindre qualité du pâturage d'automne nécessitaient vraisemblablement une plus grande recherche et tri de l'herbe, qui ont entraîné une dispersion plus grande des animaux. Le troupeau a occupé toute la surface de cette " montagne " en 3 jours en juillet et I jour en octobre.

Ces observations sont sensiblement diffétentes de celles réalisées par HUNTER (I963) en Écosse sur mouton de race Cheviot. Comme dans le cas des moutons, les vaches étaient bien groupées la nuit et un peu plus dispersées le jour ; mais elles restaient en un seul troupeau lors du pâturage et étaient plus groupées au printemps qu'à l'automne, à l'inverse des moutons observés par HUNTER.

\section{2. - Localisation des animaux et zone de végétation}

Nous avons noté sur $\mathrm{M}_{3}$ les zones de végétation avec les principales espèces dominantes. Nous avons ainsi pu calculer le temps d'occupation relatif de ces zones, exprimé par le rapport :

$$
\left(\frac{\text { temps passé sur une zone }}{\text { surface de cette zone }}: \frac{\text { temps journalier }}{\text { surface de la montagne }}\right) \times \text { roo. }
$$

Le tableau 6 montre clairement qu'en juillet les animaux ont séjourné plus fréquemment (relativement à la surface) sur les zones voisines du buron, autrefois fertilisées par le déplacement du parc de traite : la zone entourant le buron fut très occupée, la butte voisine, quoique bien fertilisée, un peu moins, peut-être parce que l'herbe y était plus souillée (aire de couchage nocturne) ; l'occupation des marécages fut très faible. Au mois d'octobre, les zones du buron, surpâturées au début de la saison, étaient délaissées au profit des autres buttes herbeuses et des marécages. Les veaux séjournaient un peu plus longtemps que les vaches dans les marécages, surtout en octobre.

Nous n'avons malheureusement pas enregistré les temps de pâturage sur les différentes zones, sauf en octobre sur les marécages ; cependant, les animaux pâturaient plus sur les buttes et pentes herbeuses que sur les zones hautes et pierreuses ou humides et marécageuses. Ces différences s'expliquent essentiellement par des différences dans la composition de la flore.

Les espèces végétales dominantes, Festuca rubra et Agrostis tenuis, étaient bien pâturées, surtout au début de la saison, mais les animaux préféraient les espèces des zones fertilisées : Poa pratensis, Trifolium repens. Taraxacum officinale, Plantago lanceolata et Phleun pratense. Achillea millefolium et Meum athamanticum présents dans toutes les zones non marécageuses et Carex praecox des limites de bas-fonds humides étaient bien pâturés. Nardus stricta abondant sur ces "montagnes " surtout sur le haut des buttes et Genistella sagittalis ( $\operatorname{sur} \mathrm{M}_{1}$ ) étaient pâturés au stade très jeune; ils étaient ensuite délaissés, comme Renonculus acer, Anemone nemorosa et les Carex et Joncus des zones marécageuses. Pourtant, les veaux broutaient parfois quelques herbes dures, ordinairement refusées par les vaches, telles que Joncus effusus, Carex 
elata et panicea, Genista (sauf Genısta anglica). Les zones marécageuses étaient moins délaissées en octobre qu'en juillet, car l'herbe y était abondante ; en $\mathrm{M}_{3}$, le temps de pâturage en zone humide s'élevait alors à I h $3^{I}$ pour les vaches et $\mathrm{I} h 5^{6}$ pour les veaux (soit respectivement 18 et $30 \mathrm{p}$. Ioo du temps de pâturage journalier) ; cela peut être important pour l'infestation des animaux par des parasites internes.

\section{TABLEAU 6}

Répartition du troupeau en fonction des zones de végétation (Montagne $\mathrm{M}_{3}$ )

\begin{tabular}{|c|c|c|c|c|c|c|c|c|}
\hline & \multicolumn{2}{|c|}{ Zone marécageuse } & \multicolumn{2}{|c|}{ Zone du buron } & \multicolumn{2}{|c|}{$\begin{array}{l}\text { Zone voisine } \\
\text { du buron }\end{array}$} & \multicolumn{2}{|c|}{$\begin{array}{l}\text { Autres buttes } \\
\text { herbeuses }\end{array}$} \\
\hline $\begin{array}{l}\text { p. } 100 \text { de la surface } \\
\text { totale } \ldots \ldots \ldots \ldots\end{array}$ & \multicolumn{2}{|c|}{31,8} & \multicolumn{2}{|c|}{3,2} & \multicolumn{2}{|c|}{10,8} & \multicolumn{2}{|c|}{54,3} \\
\hline Quantité d'herbe .... & \multicolumn{2}{|c|}{ Abondante } & \multicolumn{2}{|c|}{ Faible } & \multicolumn{2}{|c|}{ Moyenne } & \multicolumn{2}{|c|}{ Moyenne } \\
\hline $\begin{array}{l}\text { Espèces végétales do- } \\
\text { minantes } \ldots \ldots \ldots \ldots\end{array}$ & \multicolumn{2}{|c|}{$\begin{array}{l}\text { Carex elata } \\
\text { Carex panicea } \\
\text { Joncus effusus }\end{array}$} & \multicolumn{2}{|c|}{$\begin{array}{l}\text { Agrostis tenuis } \\
\text { Poa pratensis } \\
\text { Festuca rubra } \\
\text { Taraxacum officinale } \\
\text { Poa annua } \\
\text { Trifolium repens }\end{array}$} & \multicolumn{2}{|c|}{$\begin{array}{l}\text { A grostis tenuis } \\
\text { Festuca rubra } \\
\text { Trifolium repens } \\
\text { Taraxacum officinale }\end{array}$} & \multicolumn{2}{|c|}{$\begin{array}{l}\text { Festuca rubra } \\
\text { Agrostis tenuis } \\
\text { Nardus stricta }\end{array}$} \\
\hline $\begin{array}{l}\text { Temps d'occupation } \\
\text { relatif * }\end{array}$ & Vaches & Veaux & Vaches & Veaux & Vaches & Veaux & Vaches & Veaux \\
\hline $\begin{array}{l}\text { En juillet (moyenne } \\
3 \text { j) ............... } \\
\text { En octobre (movenne }\end{array}$ & 49,5 & 56,6 & $28:, 1$ & 293,8 & 152,7 & 138,9 & 108,1 & 106,3 \\
\hline 2 j) $\ldots \ldots \ldots \ldots$ & 55,2 & 67,9 & 93,8 & $59,,^{\prime}$ & 37,1 & 31,5 & 139,0 & 134,5 \\
\hline Valeur moyenne & 52,3 & 62,2 & 189,1 & 176,6 & $9_{1}^{\prime}, 9$ & 85,2 & 123,5 & 120,4 \\
\hline
\end{tabular}

* Temps d'occupation relatif $=\frac{\text { temps passé dans la zone / temps d'observations }}{\text { surface de la zone } / \text { surface de la montagne }} \times \mathbf{1 0 0}$.

\section{DISCUSSION ET CONCLUSION}

L'emploi du temps des troupeaux de vaches-mères dans l'Aubrac est comparable à celui qui a été observé par les autres auteurs dans des régions de climat tempéré (JOHNSTONE-WALLACE et KenNEDY, I944; WALKER, I962 ; HANCOCK, I953). L'activité des animaux y est cyclique, même pour les veaux; elle est essentiellement diurne, débute un peu avant le lever et se termine peu après le coucher du soleil.

Le temps de pâturage des vaches paraît déterminé par deux facteurs principaux: il augmente quand la quantité d'herbe présente diminue (avancement en saison ou moins bonne qualité du pâturage) ; il diminue quand la longueur du temps diurne disponible décroît (saison ou réduction par la traite), bien que la proportion de temps 
passé à pâturer augmente. La réduction du temps disponible ou la diminution de la quantité d'herbe diminue le nombre et allonge la durée des repas. Lorsque les conditions métérologiques sont très mauvaises, les animaux s'arrêtent de pâturer, mais pâturent ensuite avec plus d'intensité.

Le temps de pâturage des veaux des vaches allaitantes augmente constamment avec l'âge ; il est égal à la moitié de celui de leurs mères vers 3 mois $\mathrm{I} / 2$ et presque identique à 7 mois. Le comportement journalier des veaux se rapproche de celui des vaches à mesure qu'ils avancent en âge. Le temps de pâturage des veaux, dans la limite du temps disponible, est d'autant plus fort qu'ils consomment moins de lait. En définitive, l'activité de pâturage des vaches et des veaux apparaît relativement plastique.

Les troupeaux de vaches allaitantes en liberté sur les pâturages ont exploité l'ensemble de la surface disponible, mais de façon différentielle, selon les zones de végétation ; ils ont préféré celles qui avaient été anciennement fertilisées par le parcage des animaux. Les éleveurs notent, d'ailleurs, que l'abandon de la traite supprime cette répartition de la fertilisation et entraîne une dégradation de la flore par le surpâturage des bonnes espèces et le développement d'espèces peu appétibles, comme le nard raide (Nardus stricta). Le cloisonnement des "montagnes " éviterait, sans doute en partie, cette dégradation de la flore.

Nos observations permettent d'interpréter, en partie, les différences de croissance des veaux selon le mode de conduite du troupeau (tab1. 7) ainsi que certains résultats

\section{TABLEAU 7}

Infuence du mode de conduite et de la qualité des "montagnes " sur la croissance des veaux

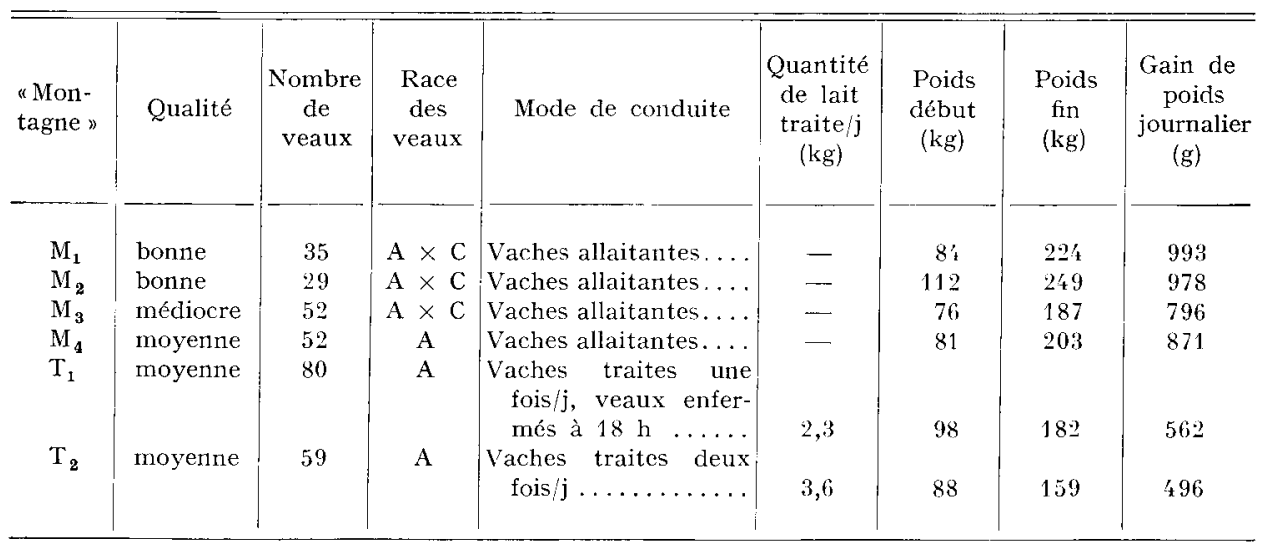

Nota : Mâles et femelles étaient en nombres voisins sur les 6 "montagnes".

obtenus par Béranger et Teissier (I970) sur d'autres "montagnes " de l'Aubrac. L'augmentation du temps de pâturage des veaux dont les mères étaient traites et qui n'étaient pas enfermés le soir $\left(\mathrm{T}_{2}\right)$, leur a permis d'avoir un gain de poids vif journalier voisin d'r $/ 2 \mathrm{~kg}$, inférieur seulement de 300 à $400 \mathrm{~g}$ à celui des veaux des vaches allai- 
tantes pâturant des montagnes de qualité comparable $\left(M_{3}\right.$ et $\left.M_{4}\right)$. Avec une quantité de lait inférieure, les veaux des vaches traites deux fois par jour ont eu une vitesse de croissance voisine de celle des veaux des vaches traites I fois par jour et qui ne pouvaient pas pâturer le soir $\left(\mathrm{T}_{1}\right)$.

BÉRANGER et TESSIER (I970) ont observé, sur deux autres "montagnes", que la croissance des veaux des vaches traites une fois par jour pouvait être améliorée d'environ Io p. Ioo, simplement en les laissant pâturer en fin d'après-midi et le soir, une parcelle de bonne qualité.

Bien que les veaux aient pâturé plus longtemps les "montagnes " de qualité médiocre, ils ont eu un gain de poids vif de $20 \mathrm{p}$. Ioo plus faible que lorsqu'ils pâturaient des " montagnes » de bonne qualité (tab1. 7). Cette plus faible croissance est bien sûr imputable en partie à la moins bonne production laitière des mères. Sur certains autres pâturages de l'Aubrac de qualité médiocre, un apport de $60 \mathrm{~kg}$ seulement d'aliment concentré a suffi à augmenter de ro p. Ioo la croissance des veaux (BÉRANGER et Tissier, I970).

Cette augmentation des quantités d'éléments nutritifs ingérés, sous forme d'herbe pâturée ou d'une quantité limitée de concentré, permet aux veaux de plus de 3 mois de se contenter d'une quantité de lait plus faible. Ils doivent avoir à leur disposition une herbe de bonne qualité pendant toute la journée.

Reçu pour publication en juillet 1971.

\section{SUMMARY}

\section{BEHAVIOUR OF COWS AND THE,IR CALVES ON THE HIGHLAND PASTURES OF AUBRAC}

The behaviour (grazing, suckling, lying down, wandering) of 6 herds composed of $29-82$ cows and their calves on the highland pastures of Aubrac (table I) has been recorded $: 4$ herds of "cows suckling their calves in freedom, $I$ herd of cows hand-milked twicc a day and $I$ herd of cows milked once a day (morning) and then on pasture with their calves until $6 \mathrm{p}$. m. (table 2). As for the latter, the calves suckled during the day and were separated from their mother at about $6 \mathrm{p} . \mathrm{m}$.

The observation method used has been the subject of a technical note (PeriT, I969).

The activity of the herds was principally diurnal and closely bound to sunrise and sunset (fig. I). However, in October, we noticed an important activity of the herd during clear nights around midnight.

The mean grazing time of the suckling cows was 8 hours per day (beginning of grazing of the first animals in the morning till the lying down of the last animals in the cvening). The grazing time was longer in August than in June-July (lowering of the quality of the pasture), but shorter in October than in August (reduction of day-timc) (table 3). The grazing time was longer in JuneJuly $(+$ I 7 per cent) on pastures of lower quality. For pastures of similar quality, the 4 to 5 hours' reduction of the time available due to milking decreased the grazing time of the cows by $\mathrm{x}$ to I I/2 hour. When the weather was bad, the cows stopped grazing, but began again more intensively either the same day or the next one (table 4).

The daily grazing time of the suckling calves gradually increased with age (table 3 , fig. 3 ). It was much longer $(+29$ per cent) on poor quality pastures. Calves of cows milked twice daily grazed, on an average, 33 minutes longer than suckling calves on pastures of similar quality; calves of cows milked once daily, and separated from them during the night, grazed 55 minutes less. This could explain the relatively small growth rate noticed in calves of cows milked once daily (table 7). Bad weather conditions do not seem to have altered the grazing time of the calves (table 4).

The reduction of the time available or decrease in the amount of grass diminished the number of grazing meals and increased their duration (tables 2 and 4 ). When getting older, the calves showed a behaviour on pasture almost similar to that of their mothers. 
The calves of suckling cows suckled for about 40 minutes per 24 hours ( 3 or times), (table 5), particularly in the morning, the evening and at the beginning of the grazing cycles. The calves of the cows milked once a day suckled during I 5 to 20 minutes when they were with their mothers.

The routes followed by the herds, during the day on the non partitioned pastures were regular, and at night, the animals always laid down on the same places. The dispersion of the herds was more important in Autumn than in the Springtime. The animals overgrazed the fertilized areas and neglected the poor ones (table 6). However, the grazing time in the marshlands was close " to I I/2 hour.

The results have been compared with those obtained by other authors and an attempt was made to interprete, from the behaviour on pasture, the growth differences in the calves according to the type of management.

\section{RÉFÉRENCES BIBLIOGRAPHIQUES}

Béranger C., Tessier J. H., ig7o. L'Aubrac, étude ethnologique, linguistique, agronomique et économique d'un établissement humain. Tome I, géographie, agronomie, sociologie économique. Paris, Éditions du Centre National de la Recherche Scientifique.

Chambers D. T., 1959. Grazing behaviour of calves reared at pasture. J. Agric. Sci., 53, 417-424.

Hancock J., r953. Grazing behaviour of cattle. Anim. Breed. Abst., 21, I-I3.

Hutchison H. G., Woof R., Habon R. M., Salehe I., RobB J. M., I962. A study of the habits of Zebu cattle in Tanganyika. J. Agric. Sci., 59, 30r-3I7.

Hunter R. F., Milner C., I963. The behaviour of individual, related and groups of south country Cheviot hill sheep. Anim. Behav, 11, 507-5 I3.

Johnstone-Wallace D. B., Kennedy K., I944. Grazing management practices and their relationship in the behaviour and grazing habits of cattle. J. Agric. Sci, 34, 190-I97.

Peterson R. A., Woolfolis E. J., 1955. Behaviour of Heveford cows and calves on short grass range. J. Range Mgmt, 8, 51-57.

Petit M., I969. Méthode d'observation de l'emploi du temps des troupeaux de vaches allaitantes au pâturage. Ann. Zootech., 18, $22 \mathrm{I}-226$.

Roy J. H. B., Shillam K. W. G., Palmer J., 1955. The outdoor rearing of calves on grass with special reference to growth rate and grazing behaviour. J. Lairy. Res., 22, 252-269.

VISSAc B., Ig7o. L'Aubrac, étude ethnologique, linguistique, agronomique et économique d'un établissement humain. Tome I, Géographie, agronomie, sociologie économique. Paris, Editions du Centre National de la Recherche Scientifique.

WAgnoN K. A., I963. Behaviour of beef cows on a california range. California. Agr. Exp. Stat. Bull., 799, $58 \mathrm{p}$.

WALKER D. E., 1962. Suckling and grazing behaviour of beef heifers and calves. N. Z. J. Agric. Res., 5, $33 \mathrm{r}-33^{8}$. 